\title{
The Anomalous Dispersion of the Disorder-Induced and the Second-Order Raman Bands in Carbon Nanotubes
}

\author{
M. A. Pimenta*1, E. B. Hanlon ${ }^{2}$, A. Marucci $^{3}$, P. Corio ${ }^{3}$, S. D. M. Brown ${ }^{3}$, \\ S. A. Empedocles ${ }^{4}$, M. G. Bawendi ${ }^{4}$, G. Dresselhaus ${ }^{5}$, and M. S. Dresselhaus ${ }^{6}$ \\ ${ }^{1}$ Departamento de Fisica \\ Universidade Federal de Minas Gerais, Belo Horizonte, 30123-970 Brazil \\ ${ }^{2}$ George R. Harrison Spectroscopy Laboratory \\ Massachusetts Institute of Technology, Cambridge, Massachusetts 02139, USA \\ ${ }^{3}$ Department of Physics, \\ Massachusetts Institute of Technology, Cambridge, Massachusetts 02139, USA \\ ${ }^{4}$ Department of Chemistry, \\ Massachusetts Institute of Technology, Cambridge, Massachusetts 02139, USA \\ ${ }^{5}$ Francis Bitter Magnet Laboratory \\ Massachusetts Institute of Technology, Cambridge, Massachusetts 02139, USA \\ ${ }^{6}$ Department of Electrical Engineering and Computer Science and Department of Physics, \\ Massachusetts Institute of Technology, Cambridge, Massachusetts 02139, USA
}

Received on 28 December, 1999

\begin{abstract}
In this work we have studied the dispersion of the disorder-induced $(D)$ and the second-order $\left(G^{\prime}\right)$ Raman bands in single wall carbon nanotubes using several laser excitation energies $\left(E_{\text {laser }}\right)$ in the range $1.5-3.0 \mathrm{eV}$. An anomalous step-like behavior was observed in the $E_{\text {laser }}$ dependence of the $G^{\prime}$-band frequency. This result is interpreted as a manifestation of the one-dimensional (1D) behavior of the phonon spectrum in carbon nanotubes.
\end{abstract}

One of the interesting features of the Raman spectrum in $s p^{2}$-bonded carbon materials is the laser energy dependence of the frequency of the disorder-induced $D$-band observed between 1250 and $1450 \mathrm{~cm}^{-1}$ and its related overtone, the second-order $G^{\prime}$-band, which appears in the range $2500-2900 \mathrm{~cm}^{-1}$ by changing the laser excitation energy from 1 to $4.5 \mathrm{eV}$. This general phenomenon is known for nearly two decades[1] and occurs in all kinds of $s p^{2}$ carbon materials, such as, graphon carbon black,[2] hydrogenated amorphous carbon,[3] glassy carbon and crystalline graphite,[4, 5, 6] and multi-component carbon films.[7] Interestingly, the second-order $G^{\prime}$ band exhibits a laser-dependent frequency even in the case of crystalline graphite, where the disorder-induced $D$ band is absent, showing that the strong dispersion of these bands is an intrinsic property of the $2 \mathrm{D}$ graphene lattice. In this work we show that the $D$ and $G^{\prime}$ bands in single wall nanotubes (SWNTs) exhibit an anomalous dispersive behavior for laser energies $\left(E_{\text {laser }}\right)$ near $2 \mathrm{eV}$, which is related to the one-dimensional (1D) character of their phonon spectrum.

Fig. 1 illustrates the basic mechanism which explains the dispersion of the $D$ and $G^{\prime}$ Raman bands $\omega\left(E_{\text {laser }}\right)$ in $s p^{2}$ carbons.[5, 6, 8] In the upper part of Fig. 1 we see that electronic transitions between the $\pi$ and $\pi^{*}$ electronic states with energies corresponding to visible photons only occur in the vicinity of the $K$ point in the Brillouin zone (BZ), where the electronic states show a linear dependence on wave vector $\mathbf{k}$. [8, 9] The phonons associated with the $D$-band and the $G^{\prime}$ band have the same wavevector $\mathbf{q}=\mathbf{k}$ as the electronic states which are in resonance with the laser. Moreover, these phonons belong to the optic branch that contains the zone center $E_{2 g_{2}}$ graphitic mode, represented by the solid curve in Fig. 1.[8, 9] The reason why the phonons belonging to this particular optic branch exhibit an especially large Raman cross-section compared to other phonons with the same wavevector $\mathbf{q}$ is attributed to their breathing-mode displacements which would be ex- 
pected to show strong deformation potential coupling to the electronic states.[8]

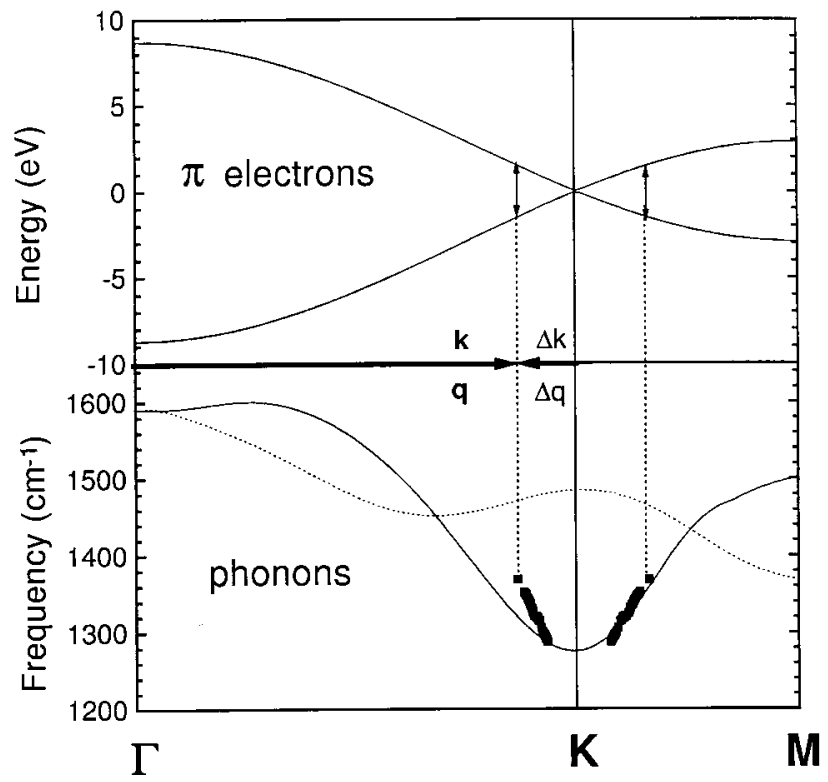

Figure 1. $\pi$ electron energy bands (top) and high frequency phonon dispersion curves of 2D graphite (bottom).[8, 9] The phonon branch that is strongly coupled to electronic bands in the optical excitation is indicated by a solid curve. The squares represent the experimental data displayed in Fig. 3, and converted according to Eqs. (1) and (2).

The Raman spectra of ordered $s p^{2}$ carbon materials generally show a second-order $G^{\prime}$-band that is much more intense than the disorder-induced $D$-band, since the $D$-band only appears when there is a breakdown in translational symmetry.[10] Therefore, a Raman investigation of the second-order $G^{\prime}$-band as a function of the energy of the incident photon provides an accurate way to probe the optic phonon branch represented by the solid curve on the bottom of Fig.1. In this work we present a detailed study of the second-order $G^{\prime}$-band of SWNTs, using several different laser energies in the range $1.5-3 \mathrm{eV}$. The analysis of the dispersive behavior of the $G^{\prime}$-band is used to investigate the phonons of SWNTs associated with this particular optic branch of 2D graphite.

The SWNT sample used in the present experiment was synthesized by the Smalley group at Rice University by laser vaporization of a carbon target containing 1 to 2 atom $\% \mathrm{Ni} / \mathrm{Co}$ in a furnace at $1200^{\circ} \mathrm{C} .[11,12]$ Details of the sample characterization are in Refs. $[11,12]$. Raman scattering experiments were performed under ambient conditions using a back-scattering geometry for the following laser excitation energy ranges and lines: Dye laser $630-540 \mathrm{~nm}$ $(1.97-2.30 \mathrm{eV})$; Ti-sapphire $820-700 \mathrm{~nm}(1.54 \mathrm{eV}$ and $1.77 \mathrm{eV})$; Krypton $647.1 \mathrm{~nm}, 568 \mathrm{~nm}$ and $406.7 \mathrm{~nm}$ $(1.92 \mathrm{eV}, 2.18 \mathrm{eV}$ and $3.05 \mathrm{eV})$; Argon $530.9 \mathrm{~nm}, 520.8$ $\mathrm{nm}, 514.5 \mathrm{~nm}, 501.7 \mathrm{~nm}, 496.5 \mathrm{~nm}, 488 \mathrm{~nm}, 476.5 \mathrm{~nm}$, $472.7 \mathrm{~nm}, 465.8 \mathrm{~nm}$ and $457.9 \mathrm{~nm}(2.34 \mathrm{eV}, 2.38 \mathrm{eV}$, $2.41 \mathrm{eV}, 2.47 \mathrm{eV}, 2.50 \mathrm{eV}, 2.54 \mathrm{eV}, 2.60 \mathrm{eV}, 2.62 \mathrm{eV}$, $2.66 \mathrm{eV}$ and $2.71 \mathrm{eV}$, respectively). The accuracy of the frequency measurements is always better than $2 \mathrm{~cm}^{-1}$.

Fig. 2 shows the second-order Raman spectra of SWNTs using three different laser energies. Note that the most prominent feature is the $G^{\prime}$-band around $2700 \mathrm{~cm}^{-1}$. The frequency of the $G^{\prime}$-band depends strongly on the laser energy, whereas the frequencies of the other second-order bands exhibit a weak laser energy dependence or no observable dependence on $E_{\text {laser }}$. A detailed analysis and discussion of the second-order spectrum of SWNTs is presented elsewhere.[13]

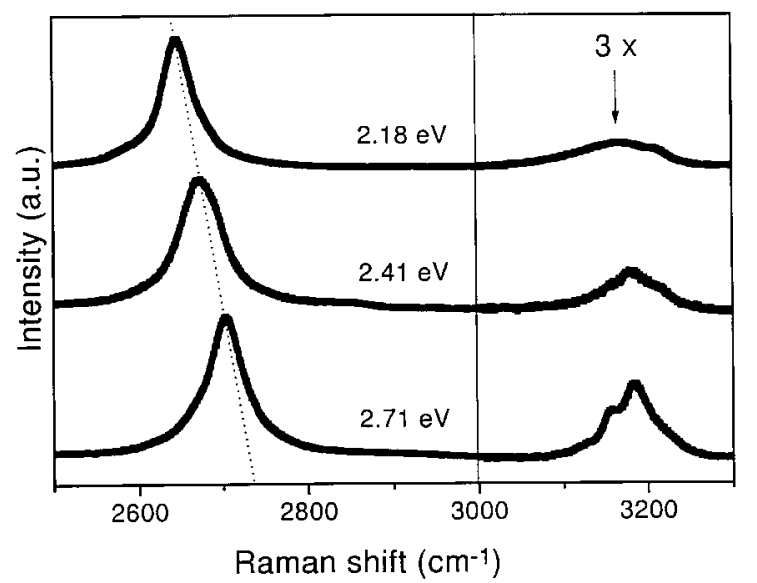

Figure 2. Second-order Raman spectra of single wall carbon nanotubes for three different laser excitation energies showing the dispersion of the $G^{\prime}$ band. The spectra above $3000 \mathrm{~cm}^{-1}$ are scaled by a factor of 3 .

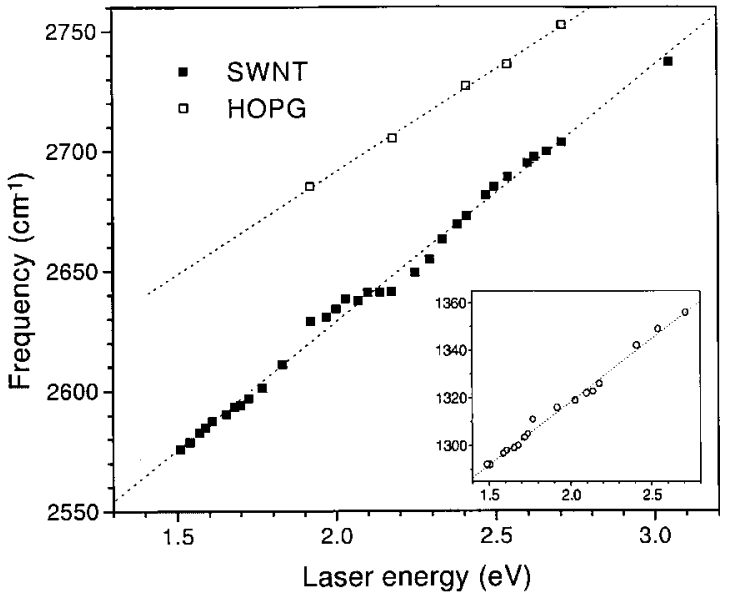

Figure 3. Dependence on the laser excitation energy of the frequency for the dominant second-order $G^{\prime}$ Raman feature for single wall carbon nanotubes (SWNT) and highly oriented pyrolitic graphite (HOPG). The inset gives the dependence of the frequency of the $D$-band on $E_{\text {laser }}$ for single wall carbon nanotubes.

Fig. 3 shows the laser energy $\left(E_{\text {laser }}\right)$ dependence of the $G^{\prime}$-band frequency $(\omega)$ for carbon nanotubes 
(SWNT). Of particular interest in Fig. 3 is the presence of an anomalous step in $\omega\left(E_{\text {laser }}\right)$ for $E_{\text {laser }}$ between 1.9 and $2.2 \mathrm{eV}$. This step is a unique behavior of SWNTs compared to all other $s p^{2}$ carbon materials, which exhibit a linear behavior for $\omega\left(E_{\text {laser }}\right)$. [1-8] The dotted line in Fig. 3 corresponds to the linear regression of the experimental data, and shows that the slope of $\partial \omega / \partial E_{\text {laser }}$ for SWNTs $\left(106 \mathrm{~cm}^{-1} / \mathrm{eV}\right)$ is slightly higher than the slopes found for other carbon materials.[1-8] The slope $\partial \omega / \partial E_{\text {laser }}$ of the $D$-band for SWNTs (see inset of Fig. 3) is $53 \mathrm{~cm}^{-1} / \mathrm{eV}$.

In order to explain the results displayed in Fig. 3, let us return to the discussion of the resonance Raman condition represented by the vertical dashed lines in Fig. 1. The phonons that contribute to the $G^{\prime}$-band for a given laser excitation energy have wavevectors $\mathbf{q}$ with the same value as the wave-vectors $\mathbf{k}$ of the electronic transitions $(\mathbf{q}=\mathbf{k})$ that are in resonance with the laser energy

$$
E_{\text {laser }}=\Delta E(\mathbf{k})
$$

Here $\Delta E(\mathbf{k})$ is the energy difference between the $\pi$ and $\pi^{*}$ electron valence and conduction bands, which for $2 \mathrm{D}$ crystalline graphite, is given by:[9]

$$
\Delta E\left(k_{x}, k_{y}\right)=2 \gamma_{0} \sqrt{1+4 \cos \frac{\sqrt{3} k_{x} a}{2} \cos \frac{k_{y} a}{2}+4 \cos ^{2} \frac{k_{y} a}{2}}
$$

in which $\gamma_{0}$ is the nearest-neighbor C-C overlap energy in $s p^{2}$ carbons and $a$ is the in-plane lattice constant (2.46 $\AA$ for graphite). Equation (1) is valid for a tightbinding Hamiltonian when we consider only nearestneighbor interactions. Equations (1) and (2) and the condition $\mathbf{q}=\mathbf{k}$ relate the laser energy $E_{\text {laser }}$ to the phonon wavevector $\mathbf{q}$ and allows us to convert the experimental data for $\omega\left(E_{\text {laser }}\right)$ to the phonon dispersion relation $\omega(\mathbf{q})$. As an example, the experimental frequencies of the $G^{\prime}$-band divided by two (since this band involves two phonons) are also plotted in Fig. 1 along the $\mathrm{K} \Gamma$ and $\mathrm{KM}$ directions of the Brillouin zone of $2 \mathrm{D}$ graphite, assuming that $\gamma_{0}=2.95 \mathrm{eV}$. [14] It must be emphasized that the $G^{\prime}$-band has contributions from all the phonons around the $K$-point which satisfy the selective resonant conditions given in Eqs. (1) and (2), thus explaining the relatively large line width of the $G^{\prime}$ band (see Fig. 2).

Because of the linear $k$ dispersion of the $\pi$ and $\pi^{*}$ bands near the $K$ point, the energy separation $\Delta E$ for small $\Delta k$ can be written [8] as $\Delta E=\sqrt{3} a \gamma_{0} \Delta k$ where $\Delta k$ is the distance between a given $\mathbf{k}$ point within the BZ and the $K$ point $\left(\Delta \mathbf{k}=\mathbf{k}_{K}-\mathbf{k}\right)$. Therefore, the slope of the laser energy dependence of the $G^{\prime}$-band frequency $\left(\partial \omega / \partial E_{\text {laser }}\right)$ can be related to the slope $\partial \omega / \partial \Delta q$ of the phonon dispersion of the $G^{\prime}$ band by

$$
\frac{\partial \omega}{\partial E_{\text {laser }}}=\frac{2}{\sqrt{3} a_{0} \gamma_{0}} \frac{\partial \omega}{\partial \Delta q} .
$$

where the factor 2 in the numerator accounts for the fact that the $G^{\prime}$-band involves two phonons. The fact that the slope $\partial \omega / \partial E_{\text {laser }}$ is slightly higher for SWNTs compared to other carbon materials can, in principle, be ascribed either to a smaller value for the overlap energy $\gamma_{0}$ or to a larger value of the phonon dispersion $\partial \omega / \partial \Delta q$. Possibly, the main contribution comes from the overlap energy $\gamma_{0}$, which is about $10 \%$ smaller for nanotubes compared to graphite [15].

Let us now discuss the step observed in $\omega\left(E_{\text {laser }}\right)$ for SWNTs between 1.9 and $2.2 \mathrm{eV}$ (see Fig. 3). Fig. 4 shows the first-order Raman spectra of SWNTs in the range $1200-1700 \mathrm{~cm}^{-1}$, in which the important features are the $D$-band in the range $1300-1350 \mathrm{~cm}^{-1}$ and the tangential band between 1500 and $1600 \mathrm{~cm}^{-1}$. Notice that the shape of the tangential band is modified when $E_{\text {laser }} \approx 2 \mathrm{eV}$. It was shown recently[14] that this effect is due to the enhancement of special Raman modes associated with the metallic nanotubes, when the laser energy lies between 1.7 and $2.2 \mathrm{eV}$ for a SWNT sample having a diameter distribution $1.37 \pm 0.18 \mathrm{~nm}$.[14]

Fig. 4 shows that the intensity of the $D$-band also increases in the range of $E_{\text {laser }}$ in which the metallic modes are enhanced. Interestingly, the frequency $\omega_{D}$ of the $D$-band practically does not change with $E_{\text {laser }}$ in this critical range of laser energies (as shown in Fig. 3, in contrast to the linear dispersion of $\omega_{D}$ observed for lower and higher laser energies. 


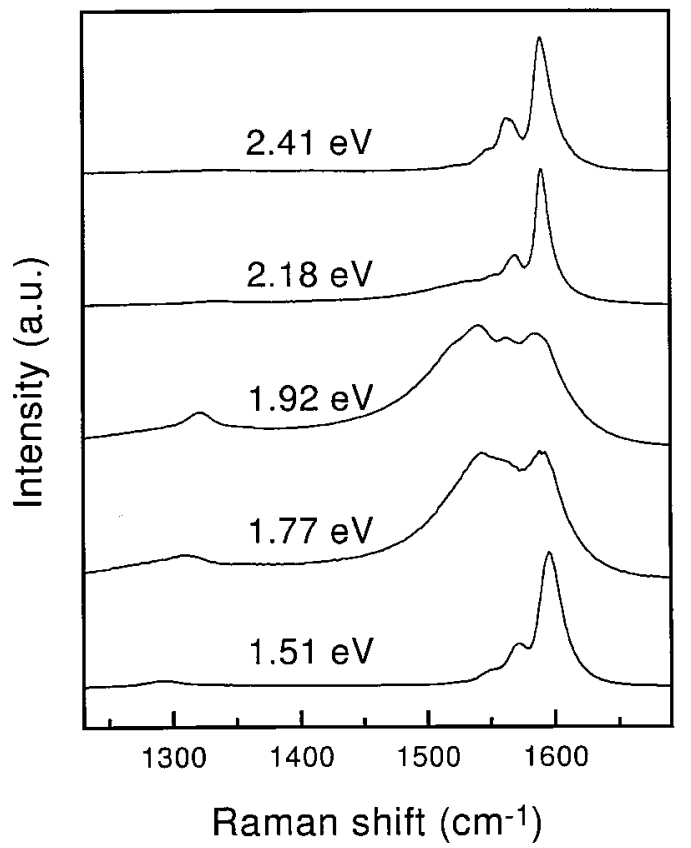

Figure 4. The first-order Raman spectra of SWNTs in the range $1200-1700 \mathrm{~cm}^{-1}$.

As already emphasized, the linear behavior of $\omega\left(E_{\text {laser }}\right)$ of the $D$-band and the $G^{\prime}$-band is a $2 \mathrm{D}$ phenomenon, common to all graphitic materials. This linear dependence on $E_{\text {laser }}$ can be explained by the model illustrated in Fig. 1. On the other hand, theoretical calculations of the vibrational spectra in SWNTs predict the existence of $1 \mathrm{D}$ phonons, that arise from the folding of the phonon dispersion relations for a 2D graphene sheet. In particular, these calculations predict the existence of Raman-active modes near $1300 \mathrm{~cm}^{-1}$, whose frequencies are expected to depend neither on the diameter of the tube, nor on $E_{\text {laser. }}[9]$

From the considerations presented above, we conclude that the $D$ and $G^{\prime}$ bands of SWNTs have two different contributions for $E_{\text {laser }}$ around $2 \mathrm{eV}$. The first contribution exhibits a linear dispersion and comes from the phonons propagating along the tube axis, with wave vectors near the $K$ point of the 2D Brillouin zone, and is described by the model presented in Fig. 1 . Similar to the case of disordered carbon materials or $s p^{2}$ carbons of small size, the Raman activity and intensity of the $D$-band in SWNTs might be associated with the finite length of the nanotubes or with the presence of defects. According to the mechanism of Fig. 1, all the SWNTs in the sample are resonantly enhanced by this process, thereby giving rise to a feature with relatively large intensity. The second contribution is non-dispersive and appears in the range of $E_{\text {laser }}$ in which the tangential modes of the metallic SWNTs are also enhanced. It comes only from the SWNTs in the sample in which the energy separation between
DOS singularities above and below the Fermi level is in resonance with the incident and/or scattered photons. This non-dispersive contribution is a manifestation of the one-dimensional behavior of the phonon spectrum in SWNTs, and is associated with the discreteness of the allowed wave vectors along the circumference of the tubes.

Finally, it is interesting to observe that the step shown in Fig. 3 for the second-order $G^{\prime}$-band is slightly upshifted in energy $(\approx 0.2 \mathrm{eV})$ with respect to the $E_{\text {laser }}$ region in which the metallic modes are enhanced in the first-order spectra. Fig. 2 also shows the overtone of the tangential band around $3200 \mathrm{~cm}^{-1}$. Just as for the first-order tangential band, the overtone band shows a corresponding line broadening when $E_{\text {laser }}$ is in resonance with metallic nanotubes. Furthermore, this overtone band is not only broadened when $E_{\text {laser }}$ for the incident beam is in resonance for metallic nanotubes but also when $E_{\text {laser }}$ for the scattered beam (which is upshifted by $\sim 0.2 \mathrm{eV}$ ) is in resonance for metallic nanotubes. Thus the $E_{\text {laser }}$ range over which the special enhanced modes appear in the second-order Stokes spectrum of the tangential modes is also upshifted by $\approx$ $0.2 \mathrm{eV}$ with respect to the critical $E_{\text {laser }}$ range in the first-order Stokes spectrum. On the basis of this comparison, we conclude that the scattered beam also plays an important role in the 1D resonant Raman effect contributing to the anomalous step behavior observed in Fig. 3 .

This work was partially supported by the Brazilian agencies FAPEMIG, CAPES and FINEP. The MIT authors acknowledge support for this work under NSF grant DMR 98-04734. The Raman measurements were performed at the George R. Harrison Spectroscopy Laboratory at MIT, supported by NIH grant P41-RR02594 and NSF grant CHE97-08265.

\section{References}

[*] e-mail: mpimenta@fisica.ufmg.br

[1] R. P. Vidano, D. B. Fishbach, L. J. Willis, and T. M. Loehr, Solid State Commun. 39, 341 (1981).

[2] T. P. Mernagh, R. P. Cooney, and R. A. Johnson, Carbon 22, 39-42 (1984).

[3] M. Ramsteiner and J. Wagner, Appl. Phys. Lett. 51, 1355 (1987).

[4] Y. Wang, D. C. Alsmeyer, and R. L. McCreery, Chem. Mater. 2, 557 (1990).

[5] A. V. Baranov, A. N. Bekhterev, Y. S. Bobovich, and V. I. Petrov, Opt. Spectrosc. USSR 62, 612 (1987).

[6] I. Pocsik, M. Hundhausen, M. Koos, and L. Ley, J. Non-Cryst. Solids 227-230 B, 1083 (1998). 
[7] B. Marcus, L. Fayette, M. Mermoux, L. Abello, and G. Lucazeau, J. Appl. Phys. 76, 3463-3470 (1994).

[8] M. J. Matthews, M. A. Pimenta, G. Dresselhaus, M. S. Dresselhaus, and M. Endo, Phys. Rev. B 59, R6585 (1999).

[9] R. Saito, G. Dresselhaus, and M. S. Dresselhaus, Physical Properties of Carbon Nanotubes (Imperial College Press, London, 1998).

[10] M. S. Dresselhaus, G. Dresselhaus, M. A. Pimenta, and P. C. Eklund. pages 367-434, Blackwell Science Ltd., Oxford, UK, 1999. Analytical Applications of Raman Spectroscopy.

[11] A. M. Rao, E. Richter, S. Bandow, B. Chase, P. C. Eklund, K. W. Williams, M. Menon, K. R. Subbaswamy, A. Thess, R. E. Smalley, G. Dresselhaus, and M. S. Dresselhaus, Science 275, 187-191 (1997), edited by M.J. Pelletier.
[12] A. Thess, R. Lee, P. Nikolaev, H. Dai, P. Petit, J. Robert, C. Xu, Y. H. Lee, S. G. Kim, A. G. Rinzler, D. T. Colbert, G. E. Scuseria, D. Tománek, J. E. Fischer, and R. E. Smalley, Science 273, 483-487 (1996).

[13] S. D. M. Brown, P. Corio, A. Marucci, M. A. Pimenta, M. S. Dresselhaus, and G. Dresselhaus, Phys. Rev. B 61, 7734 (2000).

[14] M. A. Pimenta, A. Marucci, S. Empedocles, M. Bawendi, E. B. Hanlon, A. M. Rao, P. C. Eklund, R. E. Smalley, G. Dresselhaus, and M. S. Dresselhaus, Phys. Rev. B 58, R16016 (1998).

[15] G. Dresselhaus, M.A. Pimenta, R. Saito, J.C. Charlier, S.D.M. Brown, P. Corio, A. Marucci, and M. S. Dresselhaus, (1999). Conference Proceedings, Michigan State University Workshop. Kluwer Academic, NY, page 275295, Edited by D. Tomanek and R.J. Embody. 